\title{
Optimization Model of Pure Electric Vehicle Battery-Swapping Dispatch Based on Transportation Problems
}

\author{
Shengdong $\mathrm{Zu}^{1}$, Lijiang $\mathrm{Sun}^{2}$ \\ ${ }^{1}$ Department of Electrical Engineering, Shanghai Dianji University, Shanghai,201306, China; \\ ${ }^{2}$ Department of Business, Shanghai Dianji University, Shanghai,201306, China;
}

\begin{abstract}
The optimization object of pure electric vehicle battery-swapping service dispatch is making the total cost to be minimum under the condition of meeting the battery-swapping demand. The battery-swapping service requires each charging station to scientifically transport the supplementary batteries of the electricity exchange station. It is a multi-objective optimization decision-making process. With the object of minimizing the total cost of one-time transportation, applying the transportation problems theory, an optimization model of the battery-swapping service between the charging station and the exchange station was established, and a typical example was analyzed. The results show that the battery-swapping service transportation line is one of the main factors affecting the cost of pure electric vehicle battery-swapping service dispatch.
\end{abstract}

\section{Introduction}

With the continuous growth of the global population and economic scale, environmental protection and energy shortages are facing severe pressure. Against this background, it is imperative to achieve energy transformation and industrial revitalization. The development of new energy sources, especially pure electric vehicles, is an innovation that reduces environmental pollution and eases energy tension.

There are three main ways of energy supply for electric vehicles: slow charging mode, which takes too long time to charge; fast charging mode, which have a large impact on the grid and affects battery life; The battery-swapping mode has the advantages of fast and convenient, avoiding the disadvantages of the slow charging mode of too long charging time and the fast charging mode affect the battery life. It is an ideal energy supply method.

At present, certain achievements have been made in the research on the electric vehicle replacement mode and the construction of replacement stations. Zhang Weihua ${ }^{[1]}$ proposed a three-tier service network through the popularization of standard batteries to build large-scale charging (discharging) power stations, medium-sized charging stations, battery replacement service outlets, and standard AC and DC charging piles. $\mathrm{Li} \mathrm{Wei}^{[2]}$ closely tracked the development of pure electric vehicles and charging and swapping facilities, and combined with the requirements of safety and technical aspects of the power grid, established a pure charging and swapping service network of pure electric vehicles with optimized layout and reasonable site. Zhou Fengquan ${ }^{[3]}$ analyzed the two operation modes of electric vehicle charging and battery-swapping in electric vehicle charging stations, discussed the specific operation process and profit method, and concluded that the operation mode with battery-swapping as the main and vehicle charging as the supplement will become the mainstream mode of the future development of China electric automobile charging station.

At present, there is still insufficient research on the dispatch of battery-swapping service (referred to as battery-swapping) for pure electric vehicles. This paper focuses on the analysis of the main factors that affect the dispatch of battery-swapping transportation, and proposes an optimized model of battery-swapping transportation dispatch for pure electric vehicles. The realization of the optimization model and the effects of the transportation problem model are analyzed in detail $^{[4]}$.

\section{Optimal model of electric vehicle transportation scheduling}

\subsection{Modeling ideas}

The object of the optimal dispatch of pure electric vehicle battery-swapping services is to minimize the total cost of one-time transportation dispatch under the premise of meeting the battery-swapping demand. The battery-swapping service requires each charging station to scientifically transport the supplementary batteries of the power station, which is a multi-objective

*Corresponding author's e-mail: 993697797@qq.com 
optimization decision-making route process.

If a battery-swapping service is provided, the battery needs to be transported from the charging station to a exchange station that provides pure electric vehicle battery-swapping. From the charging station, the mileage of each transportation line and the number of replacement stations passed are different. For this reason, in order to meet the battery quantity demand of daily exchange stations and reduce transportation costs, it is necessary to optimize the battery-swapping transportation route. Several primary charging stations in a certain area are sent to several secondary exchange stations, which have the characteristics of transportation problems. This article takes the example of one transportation vehicle in each line of A charging station and $\mathrm{B}$ exchange station in a certain area.

\subsection{Model establishment}

This model is to abstract the mathematical model of the actual geographical location of the charging station and exchange station design. Due to the consideration of some key factors such as economy, safety and convenience of the charging station and exchange station $^{[5]}$, the final decision was made. Site selection.

The mapping of the basic concepts of transportation is as follows:

1) Distance: The distance to be transported from each charging station to each exchange station is denoted by L.

(1) Transportation cost per kilometer: The cost per kilometer of the travel of the distribution vehicle, denoted by $\mathrm{R}$.

(2) The number of batteries delivered by each charging station is represented by $A_{x}$.

(3) The demand for the number of batteries in each exchange station is expressed in $\mathrm{B}_{\mathrm{x}}$.
(4) Objective function. The objective function of the optimal dispatch of pure electric vehicle battery-swapping services transportation is:

$$
\begin{aligned}
& \min Z=C_{11} X_{11}+C_{12} X_{12}+\cdots+C_{1 n} X_{1 n} \\
& +C_{21} X_{21}+C_{22} X_{22}+\cdots+C_{2 n} X_{2 n} \\
& \cdots \cdots \\
& +C_{m 1} X_{m 1}+C_{m 2} X_{m 2}+\cdots+C_{m n} X_{m n} \\
& =\sum_{j=1}^{n} C_{1 j} X_{1 j}+\sum_{j=1}^{n} C_{2 j} X_{2 j}+\cdots+\sum_{j=1}^{n} C_{m j} X_{m j} \\
& =\sum_{i=1}^{m} \sum_{j=1}^{n} C_{i j} X_{i j}
\end{aligned}
$$

2) Constraints. Consider the restrictions such as the daily delivery volume of the charging station and the daily demand of the exchange station. The optimization of transportation dispatch of pure electric vehicles must meet the following constraints:

(1) The number of transportation should not be greater than the number of batteries sent by the charging station: $\sum_{j=1}^{n} X_{i j} \leq a_{i}(i=1,2 \cdots m)$

(2) The number of swap batteries should not be greater than the number of transport batteries: $\sum_{i=1}^{m} X_{i j} \leq b_{j}(1,2 \cdots n)$

(3) $X_{i j} \geq 0$

Organize the data into a table, as shown in Table 1-1 below.

Table 1-1 The basic form of the table

\begin{tabular}{cccccccccccc}
\hline & & \multicolumn{1}{c}{ EV exchange station } & \multicolumn{4}{c}{ The distributing } \\
& & & $\mathrm{B}_{1}$ & $\mathrm{~B}_{2}$ & $\mathrm{~B}_{3}$ & $\mathrm{~B}_{4}$ & $\mathrm{~B}_{5}$ & $\mathrm{~B}_{6}$ & $\mathrm{~B}_{7}$ & $\mathrm{~B}_{8}$ & \multicolumn{4}{c}{ number } \\
\hline EV & $\mathrm{A}_{1}$ & $\mathrm{C}_{11}$ & $\mathrm{C}_{12}$ & $\mathrm{C}_{13}$ & $\mathrm{C}_{14}$ & $\mathrm{C}_{15}$ & $\mathrm{C}_{16}$ & $\mathrm{C}_{17}$ & $\mathrm{C}_{18}$ & $\mathrm{a}_{1}$ \\
charging & $\mathrm{A}_{2}$ & $\mathrm{C}_{21}$ & $\mathrm{C}_{22}$ & $\mathrm{C}_{23}$ & $\mathrm{C}_{24}$ & $\mathrm{C}_{25}$ & $\mathrm{C}_{26}$ & $\mathrm{C}_{27}$ & $\mathrm{C}_{28}$ & $\mathrm{a}_{2}$ \\
station & $\mathrm{A}_{3}$ & $\mathrm{C}_{31}$ & $\mathrm{C}_{32}$ & $\mathrm{C}_{33}$ & $\mathrm{C}_{34}$ & $\mathrm{C}_{35}$ & $\mathrm{C}_{36}$ & $\mathrm{C}_{37}$ & $\mathrm{C}_{38}$ & $\mathrm{a}_{3}$ \\
The quantity demanded & $\mathrm{b}_{1}$ & $\mathrm{~b}_{2}$ & $\mathrm{~b}_{3}$ & $\mathrm{~b}_{4}$ & $\mathrm{~b}_{5}$ & $\mathrm{~b}_{6}$ & $\mathrm{~b}_{7}$ & $\mathrm{~b}_{8}$ & \\
\hline
\end{tabular}

Note: $C_{\mathrm{ij}}$ is the total cost of transportation of pure electric vehicle batteries from $\mathrm{A}_{\mathrm{i}}$ to $\mathrm{B}_{\mathrm{j}}$. The calculation formula is:

$$
C_{i j}=L \times R
$$

Suppose the transportation volume from $A_{i}$ to $B_{j}$ is $X_{i j}$

\section{Example analysis}

\subsection{Brief description of the calculation example}

In order to verify the application effect of this model, the calculation example is as follows.

(1) It is known from actual investigation that every charging station and exchange station can be transported, and the actual distance $\mathrm{L}$ is measured, and the transportation cost per kilometer $\mathrm{R}=2$, as shown in Table 2-1

Table 2-1 Distance $L$ from $A_{i}$ to $B_{j}$

\begin{tabular}{lllllllll}
\hline $\mathrm{L}$ & $\mathrm{B}_{1}$ & $\mathrm{~B}_{2}$ & $\mathrm{~B}_{3}$ & $\mathrm{~B}_{4}$ & $\mathrm{~B}_{5}$ & $\mathrm{~B}_{6}$ & $\mathrm{~B}_{7}$ & $\mathrm{~B}_{8}$ \\
\hline $\mathrm{A}_{1}$ & 2 & 4 & 5 & 6.5 & 7.5 & 7 & 3 & 3.5 \\
$\mathrm{~A}_{2}$ & 5 & 6 & 5.5 & 4 & 6 & 8 & 7.5 & 3 \\
$\mathrm{~A}_{3}$ & 5 & 5.5 & 7 & 3 & 1.5 & 2 & 5 & 2 \\
\hline
\end{tabular}

(2) Assuming that the batteries transported to the exchange station just meet the demand, there is no surplus or shortage.

(3) The number and cost of batteries transported from the charging station to the exchange station on a typical day are shown in Table 2-2. 
Table 2-2 Battery transportation at each site

\begin{tabular}{cccccccccccc}
\hline & & \multicolumn{1}{c}{ EV exchange station } & \multicolumn{4}{c}{ The distributing } \\
& & & $\mathrm{B}_{1}$ & $\mathrm{~B}_{2}$ & $\mathrm{~B}_{3}$ & $\mathrm{~B}_{4}$ & $\mathrm{~B}_{5}$ & $\mathrm{~B}_{6}$ & $\mathrm{~B}_{7}$ & $\mathrm{~B}_{8}$ & \multicolumn{2}{c}{ number } \\
\hline EV & $\mathrm{A}_{1}$ & 4 & 8 & 10 & 13 & 15 & 14 & 6 & 7 & 100 \\
charging station & $\mathrm{A}_{2}$ & 10 & 12 & 11 & 8 & 12 & 16 & 15 & 6 & 150 \\
& $\mathrm{~A}_{3}$ & 10 & 11 & 14 & 6 & 3 & 4 & 10 & 4 & 150 \\
\multicolumn{2}{l}{ The quantity demanded } & 35 & 55 & 60 & 85 & 45 & 40 & 35 & 45 & \\
\hline
\end{tabular}

Solve the above transportation dispatch optimization problem.

\subsection{Example results}

Table 2-3 Base conditions of transportation scheduling

\begin{tabular}{|c|c|c|c|c|c|c|c|c|c|c|c|}
\hline & \multicolumn{9}{|c|}{ EV exchange station } & \multirow{2}{*}{$\begin{array}{c}\text { The distributing } \\
\text { number }\end{array}$} \\
\hline & & & $\mathrm{B}_{1}$ & $\mathrm{~B}_{2}$ & $\mathrm{~B}_{3} \quad \mathrm{~B}_{4}$ & $\mathrm{~B}_{5}$ & $\mathrm{~B}_{6}$ & $\mathrm{~B}_{7}$ & \multicolumn{2}{|c|}{$\mathrm{B}_{8}$} & \\
\hline $\mathrm{EV}$ & $\mathrm{A}_{1}$ & 35 & 55 & 10 & & & & & & & 100 \\
\hline charging station & $\mathrm{A}_{2}$ & & & 50 & 65 & & & & 35 & & 150 \\
\hline & $\mathrm{A}_{3}$ & & & & 20 & 45 & 40 & & & 45 & 150 \\
\hline The quantity dem & ded & 35 & 55 & 60 & 85 & 45 & 40 & & 35 & 45 & \\
\hline
\end{tabular}

$$
\begin{aligned}
\min Z=\sum_{i=1}^{m} \sum_{j=1}^{n} C_{i j} X_{i j} & =35 \times 4+55 \times 8+10 \times 10+50 \times 11+65 \times 8+35 \times 15+20 \times 6 \\
& +45 \times 3+40 \times 4+45 \times 4=2870
\end{aligned}
$$

Table 2-4 Transportation scheduling optimization results

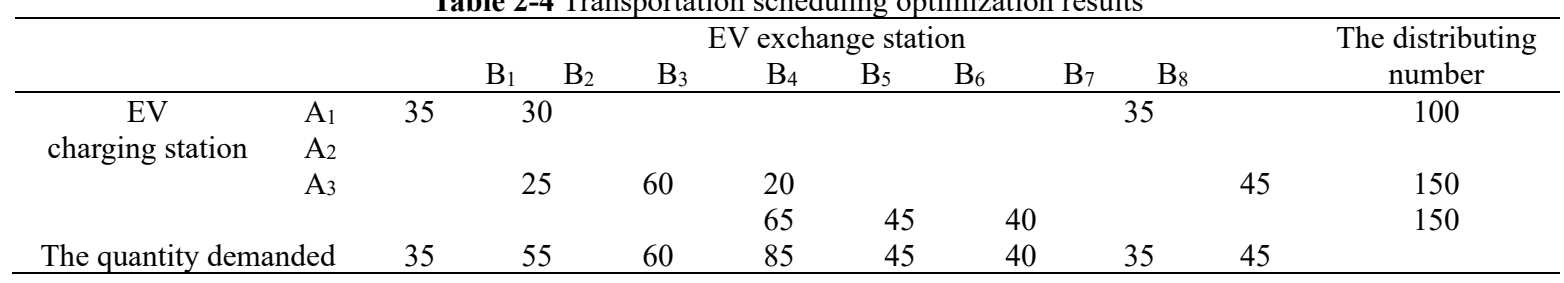

$$
\begin{aligned}
\min Z=\sum_{i=1}^{m} \sum_{j=1}^{n} C_{i j} X_{i j} & =35 \times 4+30 \times 8+60 \times 11+12 \times 25+35 \times 6+20 \times 8+45 \times 6 \\
& +65 \times 6+45 \times 3+40 \times 4=2665
\end{aligned}
$$

\subsection{Comparative analysis of results}

Before the optimization of transportation dispatch, the minimum element method is used for transportation, which has the traditional value of transportation experience. After optimizing the transportation dispatch, the Vogel method is used for transportation dispatch, and the transportation distribution route is optimized. There are calculation examples to know that the transportation cost after optimization is lower than the transportation cost before optimization, and the purpose of optimization is achieved.

\section{Conclusion}

The pure electric vehicle battery-swapping service is refined into an optimization problem. With the object of minimizing the total cost of one-time transportation, the optimization model of the battery-swapping service is established by applying transportation problem theory, and a comparative analysis is carried out in conjunction with calculation examples. The calculation results show that the battery-swapping transportation line is one of the main factors affecting the strategy of pure electric vehicle battery-swapping dispatch. This model has certain practical value in transportation dispatch, and can be used to guide the transportation route planning of battery-swapping services.

\section{References}

1. Zhang Weihua. Electric vehicle charging and replacement of the battery service network constitute research [D]. Beijing: North China Electric Power University, 2012: 8-20

2. Li Wei. Development plan of liaocheng electric vehicle intelligent charging and replacing service network [D]. Jinan: Shandong University, 2018:12-34

3. Zhou Fengquan, Lian Zhanwei, Wang Xiaolei. Research on the operation mode of electric vehicle charging station $[\mathrm{J}]$. Power System Protection and Control, 2010, 38(21): 63-66 
4. Li Xuekun. Optimization Model of Electric Vehicle Battery-Swapping Dispatch Based on Dynamic Programming [J]. Low Voltage Device, 2013, 38(16): 58-61
5. Feng Yao. Research on the key technologies of electric vehicle charging stations deployment planning [D]. Shanghai: Shanghai Jiaotong University, 2019: 48-60 